\title{
COMBO-17 measurements of the effect of environment on the type-dependent galaxy luminosity function
}

\author{
S. Phleps ${ }^{1,2}$, C. Wolf ${ }^{3}$, J. A. Peacock ${ }^{1}$, K. Meisenheimer ${ }^{4}$, and E. van Kampen ${ }^{5}$ \\ 1 Institute for Astronomy, University of Edinburgh, Royal Observatory, Blackford Hill, Edinburgh EH9 3HJ, UK \\ e-mail: sphleps@mpe.mpg . de \\ 2 Max-Planck-Institut für Extraterrestrische Physik, Giessenbachstraße, 85748 Garching, Germany \\ 3 Department of Physics, University of Oxford, Denys Wilkinson Building, Keble Road, Oxford OX1 3RH, UK \\ 4 Max-Planck-Institut für Astronomie, Königstuhl 17, 69117 Heidelberg, Germany \\ 5 Institut für Astrophysik, Leopold-Franzens-Universität Innsbruck, Technikerstraße 25, 6020 Innsbruck, Austria
}

Received 1 December 2006 / Accepted 16 March 2007

\begin{abstract}
We have developed a method to calculate overdensities in multicolour surveys, facilitating a direct comparison of the local density contrast measured using galaxy samples that have different redshift error distributions, i.e. for red and blue, or bright and faint galaxies, respectively. We calculate overdensities in small redshift slices $(\Delta z=0.02$, which at $z=0.3$ corresponds roughly to $\left.\Delta r_{\text {comoving }}=53 h^{-1} \mathrm{Mpc}\right)$ for 9176 galaxies with $R \leq 23.65, M_{B}$ (Vega) $-5 \log h \leq-18$, and $z \leq 0.7$, in three COMBO-17 fields (measuring $31^{\prime} \times 31^{\prime}$ each). The mean redshift errors of this sample are approximately $\sigma_{z} /(1+z) \simeq 0.015$. In the Chandra Deep Field South we identify a region that is underdense by almost a factor 2 compared to the other two fields in the same redshift range $(0.25 \lesssim z \lesssim 0.4)$. This can be used for an investigation of the variation of the colour-dependent luminosity function with environment: We calculate the luminosity function in this redshift range for red sequence and blue cloud galaxies (as defined by Bell et al. 2004) in each of the fields separately. While the luminosity function of the blue galaxies remains unaffected by different density contrasts, the luminosity function of the red galaxies clearly has a more positive faint-end slope in the Chandra Deep Field South as compared to the other two COMBO-17 fields. The underdensity there is thus mainly due to a deficiency of faint red galaxies. This result is in qualitative agreement with the trends seen at $z=0.1$, e.g. in the 2dFGRS (Croton et al. 2005), or in the SDSS (Zandivarez et al. 2006).
\end{abstract}

Key words. cosmology: large-scale structure of Universe - galaxies: evolution

\section{Introduction}

It has been known for more than 25 years that in a statistical sense galaxy properties depend on the local environment: there is a clear trend for early-type systems to concentrate in high-density regions (Dressler 1980; Dressler et al. 1997). This dependence on environment must hold important information about the history of galaxy formation, so it is important to study the connection between the properties of the galaxies and local galaxy density in greater detail.

The type-dependent luminosity function (the luminosity function calculated for different galaxy types) directly quantifies how the colours and luminosities of galaxies are influenced by their environment. With the advent of deep redshift surveys, it has become feasible to measure the luminosity function for field and cluster galaxies respectively (e.g. Valotto et al. 1997; Ratcliffe et al. 1998; Trentham 1998; Marinoni et al. 1999; Ramella et al. 1999; Trentham \& Tully 2002; Martínez et al. 2002; Christlein \& Zabludoff 2003; Zandivarez et al. 2006); eventually, statistical power reached the stage where the typedependent luminosity function could be estimated directly in regions of differing density contrast (e.g. Bromley et al. 1998; Gray et al. 2004).

To date, the most comprehensive analysis of this type has been that of Croton et al. (2005), who used the 2dFGRS data (Colless et al. 2003) to investigate luminosity functions split by galaxy colour in different environments. Since the $2 \mathrm{dFGRS}$ galaxies have spectroscopic redshifts, it was feasible to calculate the local density contrast in spheres of $8 h^{-1} \mathrm{Mpc}$ radius, and since the number of galaxies and the observed dynamic range of overdensities is large, the sample could be divided into six bins of density contrast.

If the dependence of galaxy properties on environment reflects the formation history of the galaxies, it is clearly of great interest to carry out similar investigations at higher redshifts. Ilbert et al. (2006a) used the VIMOS-VLT Deep Survey (VVDS; see Le Fèvre et al. 2005) to measure the environmental dependence of the total luminosity function in a sample of 6582 galaxies with spectroscopic redshifts below $z<1.5$. They also investigated the galaxy luminosity function per morphological type up to $z=1.2$ (Ilbert et al. 2006b), per spectral type up to $z=1.5$ (Zucca et al. 2006), and the build-up of the colour-density relation (Cucciati et al. 2006).

Cooper et al. (2006a) used a larger sample of 19464 DEEP2 (Davis et al. 2003) galaxies with spectroscopic redshifts in the range $0.4 \leq z \leq 1.35$. They measured the evolution of the colourdensity relation and found the fraction of red galaxies to depend strongly on environment out to $z \simeq 1$. They also investigated the type-dependent luminosity function, and found a good general agreement between their measurement and the COMBO-17 data from Wolf et al. (2004), but did not split the sample by environment (Faber et al. 2006; Willmer et al. 2006), which is explored in the present paper.

Compared to spectroscopic redshift surveys, multicolour photometric redshift surveys have generally larger number 
statistics and completeness to greater depths, and hence can be used to measure the dependence of the luminosity function on environment at intermediate redshift $(z \lesssim 1.2)$ in greater statistical detail - provided the influence of the redshift inaccuracy on the measurement of the overdensities is well understood. Using the COMBO-17 survey (Wolf et al. 2004), we will demonstrate in this paper how local overdensities can be computed and how the influence of the redshift errors can be treated. We will then show how the dependence of the (Schechter) luminosity function on the environment can be investigated, and present the results.

This paper is structured as follows: the dataset used in our study (COMBO-17) is introduced in Sect. 2. In Sect. 3 we describe the influence of redshift inaccuracies on the measurement of the local density contrast using a COMBO-17 mock catalogue, which is based on an $N$-body simulation combined with a semi-analytic model for galaxy evolution (van Kampen et al. 1999, 2005). Following this exercise, we calculate the local density contrast in three COMBO-17 fields and measure the luminosity function for red sequence and blue cloud galaxies (as defined in Bell et al. 2004) in the redshift range $0.25 \leq z \leq 0.4$ (see Sect. 4). The results are discussed in Sect. 5, and a brief summary and an outlook is given in Sect. 6 .

We assume a flat cosmological geometry with $\Omega_{\mathrm{m}}=$ 0.25 ; all lengths quoted are in comoving units, and $h=$ $H_{0} / 100 \mathrm{~km} \mathrm{~s}^{-1} \mathrm{Mpc}^{-1}$. All magnitudes are quoted with a Vega zero point.

\section{Database: the COMBO-17 Survey}

To date, COMBO-17 (Classifying Objects with Medium Band Observations in 17 filters) has surveyed three disjoint $\sim 31^{\prime} \times$ $30^{\prime}$ southern equatorial fields (the Chandra Deep Field South [CDFS], A901 and S11 field, respectively; for their coordinates see Wolf et al. 2003, W03 in the following) to deep limits in 5 broad and 12 medium passbands, covering wavelengths from 400 to $930 \mathrm{~nm}$. The classification is reliable for $R \lesssim 24$. A detailed description of the survey along with filter curves can be found in Wolf et al. (2004).

Galaxies were detected on the deep $R$-band images by using SExtractor (Bertin \& Arnouts 1996). The spectral energy distributions (SEDs) for $R$-band detected objects were measured by performing seeing-adaptive, weighted-aperture photometry in all 17 frames at the position of the $R$-band detected object.

\subsection{Photometric redshifts}

Using the 17-band photometry, objects are classified using a scheme based on template spectral energy distributions (SEDs) (Wolf et al. 2001b,a). Each object is also assigned a redshift (if it is not classified as a star). The redshift errors in this process depend on the magnitude and type of the object. The galaxy redshift estimate quality has been tested by comparison with spectroscopic redshifts for almost 1000 objects (see Wolf et al. 2004). At bright limits $R<20$, the redshift errors are approximately $\epsilon_{z} /(1+z) \simeq 0.01$, and the error is dominated by mismatches between template and real galaxy spectra. This error can contain a systematic component that is dictated by the exact filter placement, but these "redshift focusing" effects are of the order of magnitude of the random redshift errors for $z<1$ and are unimportant for the current analysis. At the median apparent magnitude $R \simeq 23, \epsilon_{z} /(1+z) \sim 0.02$. For the faintest galaxies, the redshift accuracy approaches those achievable using traditional broadband photometric surveys, $\epsilon_{z} /(1+z) \gtrsim 0.05$.
We thus restricted our analysis to galaxies with $R<23.65$. In order to define a volume limited sample at $z \leq 0.7$, we furthermore select galaxies with restframe $B$-band magnitudes $M_{B}-5 \log _{10} h \leq-18.0$, and find 9176 galaxies with $z \leq 0.7$ that fulfil these requirements. There is no point in trying to correct for the incompleteness at high redshifts, because any completeness correction requires the knowledge of the luminosity function as a function of galaxy type, which is not determined accurately enough, so we investigate the local density contrast only at $z \leq 0.7$.

\subsection{Red and blue galaxies}

From the COMBO-17 data we know the redshift and the SED for each galaxy, so it is possible to calculate their absolute restframe magnitudes and colours. We can use this information to investigate the properties of different galaxy types, e.g. red sequence and blue cloud galaxies, where we use the prescription of Bell et al. (2004) to separate the two populations from each other:

Red sequence: $(U-V)>(U-V)_{\lim }$

Blue cloud: $\quad(U-V)<(U-V)_{\lim }$

$(U-V)_{\lim }=1.25-0.4 z-0.08\left(M_{V}-5 \log _{10} h+20\right)$,

where $z$ denotes the redshift of a given galaxy.

The observed bimodality in the colour-magnitude plane permits a model-independent definition of the two different galaxy populations. While the blue cloud consists of late-type, starforming galaxies, the well defined red sequence contains mainly early-type quiescent galaxies.

\section{Method}

The local density contrast

$\delta=\frac{\rho-\bar{\rho}}{\bar{\rho}}$

where $\rho$ is the local density, and $\bar{\rho}$ the mean density, is commonly measured in spheres with a radius of e.g. $r=8 h^{-1} \mathrm{Mpc}$, as adopted by Croton et al. (2005).

Obviously, the redshift errors in our sample are too large to calculate overdensities in such spheres, but it is still possible to measure overdensities, albeit in slightly larger volumes. Instead of counting the number of galaxies in a sphere or a cylinder centred on individual galaxies, or distributed randomly within the survey volume, we calculated the comoving space densities $\rho(z)$ in redshift bins of $\Delta z=0.02$ and steps of $\delta z=0.005$. At a redshift of $z=0.3$, this corresponds to to a comoving radial bin size distance of $\Delta r \simeq 53 h^{-1} \mathrm{Mpc}$. The width of one COMBO-17 field at that redshift $\left(30^{\prime}\right)$ is approximately $7.4 h^{-1} \mathrm{Mpc}$.

This measurement cannot be compared directly with those where the overdensities are estimated in spheres $\left(r=8 h^{-1} \mathrm{Mpc}\right.$ for Croton et al. 2005 or $r=5 h^{-1} \mathrm{Mpc}$ for Cucciati et al. 2006): in our case the comoving distance along the line of sight is more than 7 times larger than the transverse distance, which is of the order of magnitude of the typical radius of the spheres. This geometry is enforced by the photometric redshift errors, and means we measure trends averaged within a larger volume. But at least this approach means we avoid uncertainties due to smallscale peculiar velocities, which complicate the measurements in smaller spheres. In the end, numerical simulations are required for a full interpretation of any of these results. 


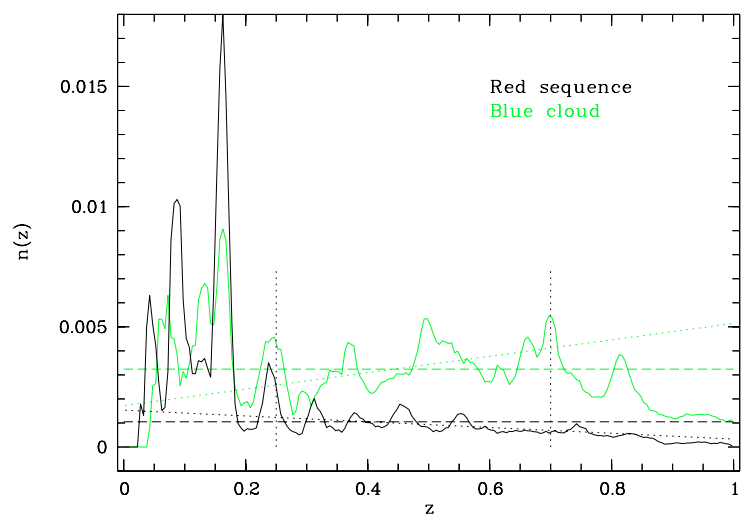

Fig. 1. The mean comoving number density of the three fields, for the blue (grey line) and red (black line) subsamples. The dotted lines show the best fits (fitted in the range $0.25 \leq z \leq 0.7$, as indicated by the vertical dotted lines) if an evolution of the number density with redshift is assumed. The dashed lines indicate the constant mean.

\subsection{Determination of the mean density}

The mean density $\bar{\rho}$ was estimated in the range $0.25 \leq z \leq 0.7$ : This avoids contamination from faint galaxies in the pre-selected cluster Abell 901 at $z=0.165$, which could be scattered in redshift due to large photometric redshift errors. Also, with a total field size of $0.78 \square^{\circ}$ and the correspondingly small survey volume at $z<0.25$, a measurement of the mean number density is always dominated by cosmic variance. Redshifts larger than $z=0.7$ are excluded, because the sample then starts to become incomplete and noisy.

Figure 1 shows the mean comoving number density (the average of the three fields), for the blue and red subsamples. While the number of red galaxies remains roughly constant with redshift, the comoving number of blue galaxies tends to increase. We fit the trend (again over the redshift range $0.25 \leq z \leq 0.7$ ) with a straight line, and use this empirical evolutionary fit instead of the constant mean in the calculation of the overdensities,

$\bar{\rho}(z)=1.72 \times 10^{-3}+3.42 \times 10^{-3} z h^{3} \mathrm{Mpc}^{-3}$.

This fit certainly overpredicts the number density at $z \geq 0.8$, so we restrict this analysis to lower redshifts. We will mainly be interested in practice in $z \simeq 0.3$, where the results have little dependence on the strength of the assumed evolution.

\subsection{Redshift errors}

Photometric redshift estimates have significant errors, and we need to understand how these affect our estimates of the density contrast. The comparison of our multicolour redshifts with the available spectroscopic ones in the CDFS (e.g. taken from the 2dFGRS and VVDS) showed no dependence of the redshift errors on the galaxy type. With 17 filters the shapes of the SEDs are very well sampled and the $4000 \AA$ break only plays a minor role in the classification and redshift estimation of the objects. The $4000 \AA$ break only becomes a problem for faint galaxies $(R \gtrsim 22)$ at the low- and high redshift end of the observations $(z \lesssim 0.15$, and $z \gtrsim 1.15$, respectively), when the break is located at the edge of the filter set (Wolf et al. 2001a). However, the redshift accuracy depends on observed magnitude: statistically, blue galaxies are fainter than red ones and thus tend to have slightly larger errors. Therefore, when comparing the properties of red sequence with those of the blue cloud galaxies, we have to test

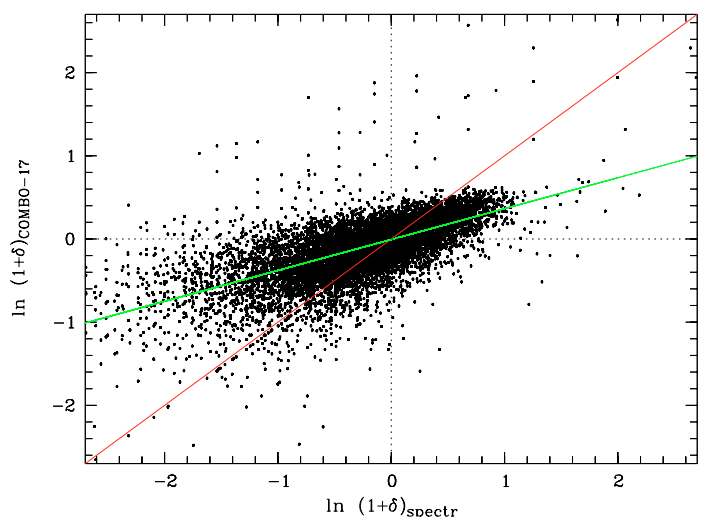

Fig. 2. The overdensities in a mock COMBO-17 survey, calculated for "spectroscopic" and "multicolour" redshifts in bins of $\Delta z=0.02(z \leq$ 0.7). A formal fit to the data points (dotted grey line) shows how the amplitudes of both over- and underdensities are diminished.

which effect the different error distributions have on the measured overdensities of our two subsamples.

In order to simulate the influence of redshift errors on the measurement of local densities in different volumes, we use a mock galaxy survey based on a set of simulations by van Kampen et al. (1999, 2005). The phenomenological model predicts positions on the sky, redshifts including peculiar velocities, magnitudes that would be measured in the COMBO-17 filters, and absolute rest-frame luminosities and colours in the same bands that we use for the analysis of the observations.

Four different simulation volumes are used to produce 80 different lightcones representing individual COMBO-17 fields, for which we also calculate the overdensities (Eq. (4)). Each of these COMBO-17 mock samples is selected in the same way as the observed data $\left(R \leq 23.65, M_{B}-5 \log _{10} h \leq-18\right.$. $)$. Their number counts and overall redshift distribution have the same expectation, but they differ in detail - thus allowing us to assess the significance of "cosmic variance".

For each galaxy in the COMBO-17 catalogue the rms error of its estimated redshift is provided by the classification scheme, and we use these errors to convolve the "spectroscopic" redshifts in our mock sample with the error distribution. For each galaxy in the mock catalogue we randomly pick out a value $\epsilon_{z}$ from the COMBO-17 data, then draw an error $\delta_{z}$ from a Gaussian distribution with $\sigma=\epsilon_{z}$, and add this error to the given redshift. Using these new, "multicolour" redshifts, we can repeat the calculation of the galaxy properties (e.g. K-corrections and rest-frame magnitudes), and of the overdensities.

Figure 2 shows the overdensities calculated for "spectroscopic" redshifts against the "multicolour" measurements in the same redshift bins. The scatter is small enough to facilitate a measurement of overdensities in a multicolour survey such as COMBO-17. However, the tilt of the relation shows that high overdensities become slightly lower, and deep underdensities slightly shallower - the dynamic range shrinks and the convolution with the redshift error distribution washes out the measured structures.

In order to facilitate a direct comparison between red sequence and blue cloud galaxies, we have to understand what effect their slightly different redshift error distributions have on the measurement, and how we can correct for any differences. We therefore now simulate redshift errors by drawing appropriate rms errors from the red sequence and blue cloud galaxy catalogues separately, and then calculate the colour-dependent 
overdensities for the mock sample. As can be seen from Fig. 4, in the presence of redshift inaccuracies the existing small scale density fluctuations are washed out, and the amplitudes of the overdensities are suppressed. Due to the statistically slightly larger errors of the blue galaxies, the signal is more strongly suppressed than when the errors of the red galaxies are applied. Thus, if we were to measure exactly the same structure using a sample of red and blue galaxies as tracers, the different redshift accuracies would cause us to infer a larger overdensity (or smaller underdensity) from the red sample than from the blue one.

To account for this, we convolve the redshift distribution of the red galaxies with a blurring function, which broadens their redshift error distribution to make it resemble the redshift error distribution of the blue galaxies. Of course the same procedure has to be applied to the bright galaxies as well, in order to make them comparable to the faint ones. In general, for each comparison we have to make sure that the redshift distribution of the sample with the smaller redshift errors has been blurred in order to make its error distribution resemble the one with the lower accuracy.

The blurring function can be found via the convolution theorem. Denote the redshift error by $\epsilon_{z}$, and let $f$ and $g$ be the redshift error distributions of red and blue galaxies respectively. We now seek a blurring function $b\left(\epsilon_{z}\right)$ that makes them compatible:

$f\left(\epsilon_{z}\right)=g\left(\epsilon_{z}\right) * b\left(\epsilon_{z}\right)$,

which is simply solved in Fourier terms: $B(k)=F(k) / G(k)$, where $k$ is a wavenumber in redshift space.

In order to evaluate the error distributions and account for its redshift dependence, we calculate in each redshift bin of size $\Delta z=0.1$ between $z=0$ and $z=1$ the sum of Gaussians where the widths $\sigma_{i}$ are the rms errors of the redshift estimated by the multicolour classification scheme (Wolf et al. 2001a, 2004):

$f\left(\epsilon_{z}\right)=\frac{1}{N_{\text {gal }}} \sum_{i=1}^{N_{\text {gal }}} \frac{1}{2 \pi \sigma_{i}^{2}} \exp \left(-\frac{\epsilon_{z}^{2}}{2 \sigma_{i}^{2}}\right)$,

for all colour and luminosity samples under consideration (for red sequence and blue cloud galaxies see Fig. 3). The resulting functions can be closely approximated by a Breit-Wigner or Lorentz curve, and it is convenient to treat this as the exact form:

$f\left(\epsilon_{z}\right)=\frac{W / 2 \pi}{\epsilon_{z}^{2}+\frac{W^{2}}{4}}$,

where $W$ is the full width at half maximum. We parameterize the evolution with redshift (in the redshift range $0.25 \leq z \leq$ 0.7 ) of the full width at half maximum $W$ with a second order polynomial:

$W(z)=a_{0}+a_{1}(1+z)+a_{2}(1+z)^{2}$,

with the following coefficients for our red and blue, and bright $\left(M_{B}-5 \log _{10} h<-19.5\right)$ and faint $\left(M_{B}-5 \log _{10} h>-19.5\right)$ subsamples. Red sequence: $a_{0}=0.015, a_{1}=0.033, a_{2}=0.026$. Blue cloud: $a_{0}=0.090, a_{1}=0.142, a_{2}=0.068$. Bright galaxies: $a_{0}=0.124, a_{1}=0.200, a_{2}=0.087$. Faint galaxies: $a_{0}=0.094$, $a_{1}=0.164, a_{2}=0.082$.

The Fourier transform of a Lorentzian is

$F(k)=\exp (-W|k| / 2)$,

from which is is readily seen that the required blurring function is

$b\left(\epsilon_{z}\right)=\frac{\Delta W / 2 \pi}{\epsilon_{z}^{2}+\frac{(\Delta W)^{2}}{4}}$,

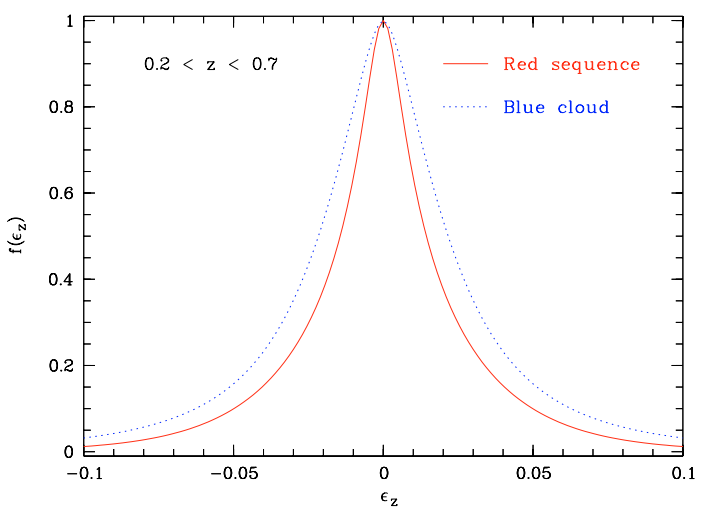

Fig. 3. The redshift error distributions of red sequence (solid line) and blue cloud galaxies (dotted line), in the redshift range $0.2 \leq z \leq 0.7$.

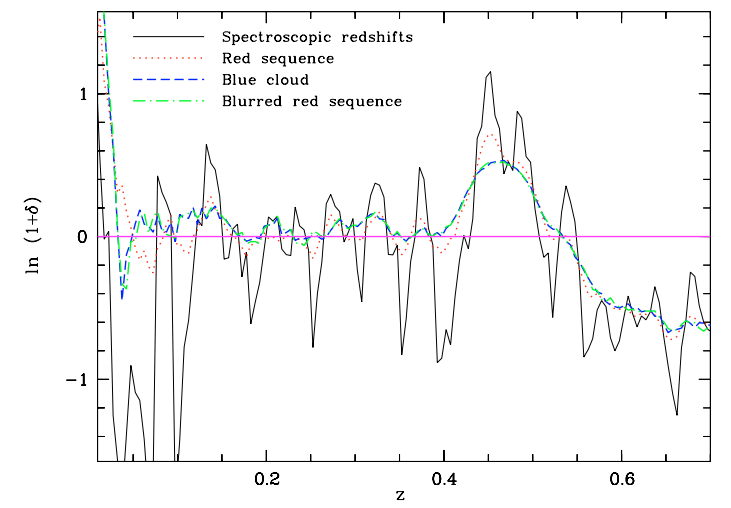

Fig. 4. Overdensity of the mock galaxies in one "COMBO-17 field". The solid line is the overdensity of the galaxies with "spectroscopic" redshifts, the dotted line is the measurement using the same galaxies, but convolved with the error distribution of the red COMBO-17 galaxies, the dashed line is the measurement using the blue COMBO-17 galaxies, and the dashed-dotted line is the overdensity of the mock galaxies, which have first been convolved with the red error distribution, and then further blurred in order to make their redshift inaccuracy comparable to the ones that have been convolved with the blue error distribution.

where $\Delta W$ is the difference in widths of the two populations. We can now use this probability distribution to degrade the redshift accuracy of a given sample in order to be comparable to another sample with larger redshift errors: a redshift offset is drawn randomly from the blurring probability distribution, and added to the true data.

Figure 4 also shows the overdensity of the mock galaxies, which have first been convolved with the red error distribution, and then further blurred in order to make their redshift inaccuracy comparable to the ones that have been convolved with the blue error distribution. Since the photometric redshift accuracies of the sub-samples are now equal by construction, we can now start to look for differences in the overdensity patterns as a function of colour or luminosity.

\section{Results}

\subsection{Overdensities in the COMBO-17 survey}

Figure 5 shows the overdensities measured in the three COMBO-17 fields, which we calculated in relatively large bins of $\Delta z=0.05$ (which corresponds to $\Delta r \simeq 132.5 h^{-1} \mathrm{Mpc}$ at $z=0.3$ ), in steps of $\delta z=0.01$. Later we will decrease the size 


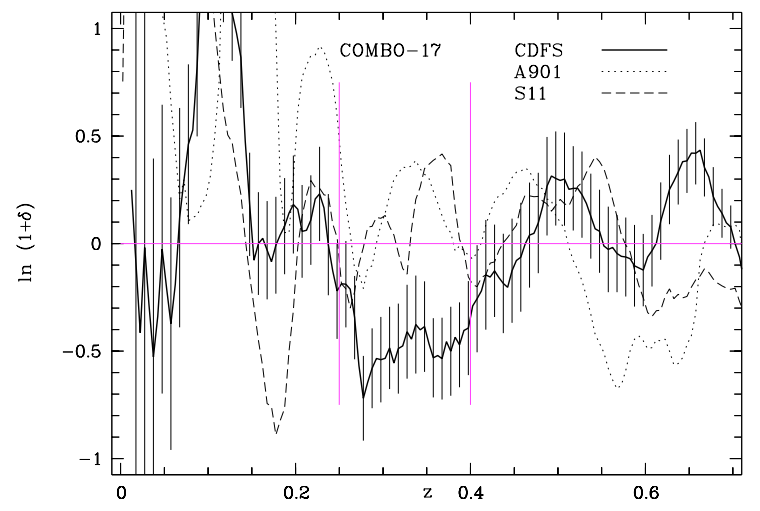

Fig. 5. The overdensities in the three COMBO-17 fields, calculated in bins of $\Delta z=0.05$ (which corresponds to $\Delta r \simeq 132.5 h^{-1} \mathrm{Mpc}$ at $z=0.3$ ) and steps of $\delta z=0.01$, versus redshift. The vertical lines indicate the range in which the luminosity function will be estimated. The errors are rms errors calculated from 80 COMBO-17 mock catalogues, but in order to avoid confusion error bars are only plotted for every second data point.

of our bins again, but here we want to compare the large-scale properties of the three fields.

The error bars plotted for the CDFS are the variances of the overdensities calculated in 80 mock COMBO-17 fields and should thus include not only Poisson noise, but also cosmic variance. However, since the data points are highly correlated (the spacing of the bins being smaller than the binsize), it should be noted that the errors are also correlated.

One well-known high-redshift structure in the CDFS, a sheet at $z=0.66$ (Gilli et al. 2003; Wolf et al. 2004; Adami et al. 2005), clearly shows up in our measurement (see Fig. 5). For the present paper, we are more interested in the range $0.25 \leq$ $z \leq 0.4$. Here one of our three fields, the CDFS, is underdense with respect to the others. The mean overdensity in the CDFS is $\delta=-0.36 \pm 0.08$, whereas in the A901 field it is $\delta=0.16 \pm 0.21$, and in the $\mathrm{S} 11$ field we find $\delta=0.11 \pm 0.24$, respectively. So in both A901 and S11 the overdensity fluctuates about the mean, whereas the CDFS is clearly underdense in this redshift range.

This is a fortunate coincidence: owing to the smaller number of galaxies and the dynamic range of overdensities observed by COMBO-17 we can not split our sample into overdensity bins in the way e.g. Croton et al. (2005) did - but we can compare the statistical properties of the galaxies in this specific underdense region with those in "normal" dense regions at the same redshift.

Before measuring and comparing luminosity functions, we calculate the overdensities in this field again for different subsamples (this time in smaller bins of size $\Delta z=0.02$ and $\delta z=$ 0.005): a sample of red sequence and blue cloud galaxies (see Sect. 2.2), and a sample of bright $\left(M_{B}-5 \log _{10} h<-19.5\right)$, and faint $\left(M_{B}-5 \log _{10} h \geq-19.5\right)$ galaxies, respectively, see Fig. 6 . The numbers of galaxies in the different subsamples are given in Table 1

Unfortunately the mock COMBO-17 catalogues we used to calculate the rms errors of the overdensities can currently not be used to calculate errors for red and blue (or bright and faint) subsamples as well, since the mock galaxies do not exhibit the same dependencies of colour and luminosity on the local density contrast as the observed galaxies. A thorough error analysis has thus to be postponed to a future paper, when improved mocks are available.

From Fig. 6, it is evident that although the redshifts of the red and bright samples have been further smoothed in the way
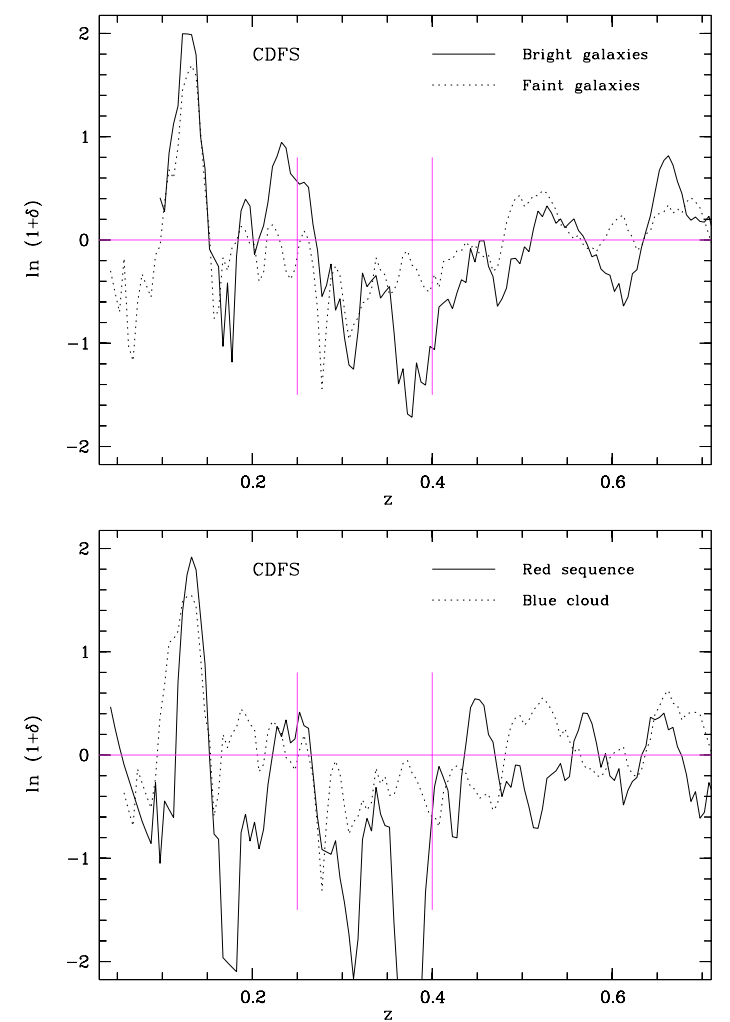

Fig. 6. The overdensities in the CDFS, calculated in bins of $\Delta z=0.02$, for bright $\left(M_{B}-5 \log _{10} h<-19.5\right)$, and faint $\left(M_{B}-5 \log _{10} h \geq-19.5\right)$ galaxies (upper panel), and red sequence and blue cloud galaxies (lower panel). For both the bright and red sequence subsamples, the redshifts have been blurred, in order to make their redshift inaccuracies similar and thus the measurement of the overdensities comparable to the faint and blue cloud subsamples, respectively.

Table 1. The numbers of galaxies in the different subsamples, per COMBO-17 field. All galaxies are preselected to have $R \leq 23.65$, $M_{B}-5 \log _{10} h \leq-18$., and $0.25 \leq z \leq 0.4$. "Bright" means $M_{B}-$ $5 \log _{10} h<-19.5$, and "faint" $M_{B}-5 \log _{10} h \geq-19.5$.

\begin{tabular}{r|rrrrr}
\hline \hline COMBO-17 field & $N_{\text {tot }}$ & $N_{\text {red }}$ & $N_{\text {blue }}$ & $N_{\text {bright }}$ & $N_{\text {faint }}$ \\
\hline CDFS & 301 & 56 & 245 & 63 & 238 \\
A901 & 594 & 162 & 432 & 165 & 429 \\
S11 & 543 & 178 & 365 & 141 & 402 \\
\hline
\end{tabular}

explained in Sect. 3, the structures are more distinct in the $\mathrm{red} /$ bright samples than in the blue/faint ones, respectively.

The different samples trace the underlying dark matter density field differently. Bright galaxies are generally found to be more strongly clustered than the faint ones, because they are thought to reside in massive dark matter haloes, which are generally believed to be more strongly clustered than small ones (e.g. Cole \& Kaiser 1989; Mo \& White 1996; Sheth \& Tormen 1999). At the same time, red galaxies are observed to be more strongly clustered than the blue galaxies (e.g. Davis \& Geller 1976; Norberg et al. 2002; Zehavi et al. 2005; Phleps \& Meisenheimer 2003; Phleps et al. 2006; Meneux et al. 2006). However, it is presently not clear whether luminosity or colour is the determining property (see e.g. Norberg et al. 2002).

As can be seen in Fig. 6, the underdensity in the CDFS at $0.25 \leq z \leq 0.4$ is particularly pronounced when calculated using only red galaxies for the determination - this region is mainly deficient in red galaxies. We will see in the next section that this 

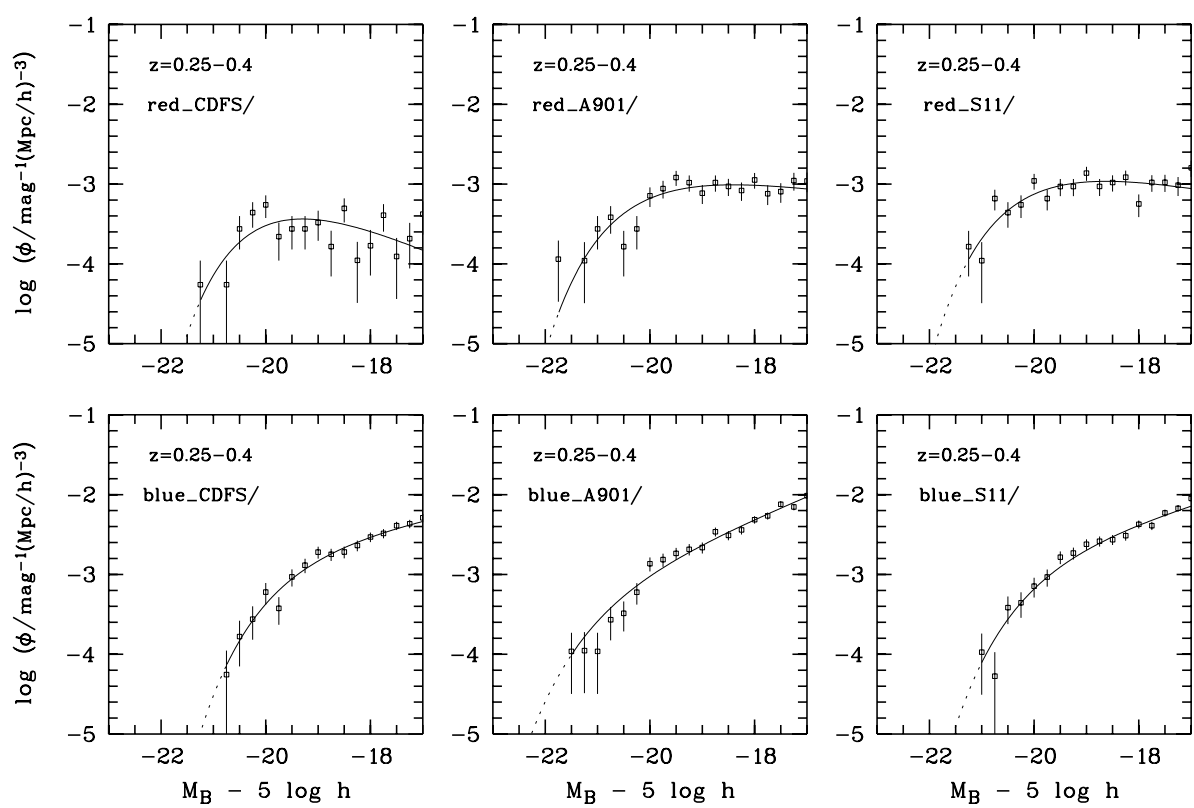

Fig. 7. The luminosity function of the red sequence (upper panel) and blue cloud (lower panel) galaxies in the redshift range $0.25 \leq z \leq 0.4$, for the three COMBO- 17 fields. The STY fit is overplotted in each panel.

Table 2. STY fit parameters for the luminosity functions of red sequence and blue cloud galaxies in the redshift range $0.2 \leq z \leq 0.4$. The numbers of the galaxies per subsample and field are given in Table 1.

\begin{tabular}{llcrc}
\hline \hline Sample & Field & $M^{*}-5 \log h($ Vega mag) & $\phi^{*} \times 10^{-4} h^{3} \mathrm{Mpc}^{-3}$ & $\alpha$ \\
\hline \multirow{3}{*}{ blue } & CDFS & $-19.53 \pm 0.33$ & $25.00 \pm 1.39$ & $-1.34 \pm 0.17$ \\
& A901 & $-20.88 \pm 0.38$ & $9.23 \pm 0.39$ & $-1.68 \pm 0.09$ \\
& S11 & $-19.89 \pm 0.31$ & $22.65 \pm 1.06$ & $-1.49 \pm 0.12$ \\
\hline \multirow{2}{*}{ red } & CDFS & $-19.64 \pm 0.41$ & $10.35 \pm 1.25$ & $-0.27 \pm 0.35$ \\
& A901 & $-20.10 \pm 0.31$ & $18.19 \pm 1.31$ & $-0.79 \pm 0.17$ \\
& S11 & $-19.97 \pm 0.28$ & $22.49 \pm 1.54$ & $-0.71 \pm 0.17$ \\
\hline
\end{tabular}

deficiency reflects mainly a reduction in the number of faint red galaxies.

\subsection{Luminosity functions}

In order to investigate which galaxies are most deficient in the underdense region in the CDFS, we we have calculated restframe $B$-band luminosity functions for the galaxies in the redshift bin $0.25 \leq z \leq 0.40$ in all three fields, split by colour according to Eq. (3).

At redshift $z=0.4$, a luminosity of $M_{B}-5 \log _{10} h=-17$ corresponds to an observed-frame apparent magnitude of $R_{\text {tot }}=$ 23.2 or $R_{\text {aper }}=23.5$ in the COMBO-17 apertures. The aperture magnitudes and colours determine the completeness, which we estimate as $>90 \%$ at every point in the redshift-luminosity data cube. Nevertheless, the calculation of the luminosity functions has been implemented exactly as described in W03 and later COMBO-17 papers, where the non-parametric $1 / V_{\max }$ estimator (Schmidt 1968) is used in the form proposed by Davis \& Huchra (1982) and modified by Fried et al. (2001), who for the first time took completeness corrections into account in the calculations. Any redshift bins where the magnitude cutoff of the survey shrinks the accessible volume of the bin by more that $30 \%$ compared to an infinitely deep survey are ignored, thus ensuring that the faint end of the luminosity function is correctly represented.

Figure 7 shows the luminosity functions of the red sequence and blue cloud galaxies in the redshift bin $0.25 \leq z \leq 0.4$ for the three COMBO-17 fields. Parameters for the Schechter fits (as plotted in Fig. 7) are given in Table 2. We present the luminosity functions separately for our three fields in order to investigate their differences. In W03 it was already reported that the CDFS is underdense in the "semi-local" redshift bin $z=[0.2,0.4]$ (see their Fig. 12). However, W03 investigated luminosity functions either split by field or split by spectral type. In contrast, here we present the LF split both by field and by rest-frame colour.

As can be seen from Fig. 7 and Table 2, the luminosity function of the blue cloud galaxies does not differ from field to field (apart from the normalisation $\phi^{*}$, which unsurprisingly is lower in a low density region). In contrast, the luminosity function of the red sequence galaxies in the CDFS (the underdense region) is indeed clearly distinct from the one measured in the two other fields, which have about mean density. Not only is the normalisation slightly lower, but also the slope $\alpha$ is clearly more positive: the underdensity in the CDFS is mainly due to a deficiency of faint red galaxies.

\section{Discussion}

Our detection of a lack of faint red galaxies in voids is in qualitative agreement with the work of Croton et al. (2005), who investigated the influence of the environment on galaxy properties in the local universe $(0.05 \leq z \leq 0.15)$ using 2 dFGRS data (Colless et al. 2001, 2003). Croton et al. were able to measure the type-dependent luminosity functions in six different overdensity regimes from voids to clusters of galaxies. They found that latetype galaxies display a consistent luminosity function across all density environments, with a weak dimming of $M^{*}$ in the underdense regions and an almost constant faint-end slope. In contrast the luminosity function of the red galaxies differs sharply 
between the extremes in environment: $M^{*}$ brightens by approximately 1.5 mag going from voids to clusters, while the faint-end slope moves from $\alpha \simeq-0.3$ in underdense regions to $\alpha \simeq-1.0$ in the densest part of the survey.

A similar analysis has been undertaken by Zandivarez et al. (2006), who investigated the variation of the galaxy luminosity function at $0.02 \leq z \leq 0.22$ with the mass of galaxy groups identified in the Fourth Data Release of the Sloan Digital Sky Survey (Adelman-McCarthy et al. 2006), and found a continuous brightening of the characteristic magnitude, and a steepening of the faint end slope as the group mass increases. When they split their sample by $u-r$ colour into red and blue galaxies, they found that the changes observed as a function of group mass are mainly seen in the red, passively evolving, galaxy population, while the luminosities of blue galaxies remain almost unchanged with mass. When we take the group mass as correlating with the local density, then this result is consistent with the result of both Croton et al. (2005), and with our own.

Therefore we conclude that the same dependency of the luminosity function on environment - a lack of faint red galaxies in underdense regions and a dominant population of bright red galaxies in overdense environments - was already in place up to $z \simeq 0.4$. We now have to ask what is known about environmental trends at higher redshifts.

Results from the DEEP2 Galaxy Redshift Survey (Davis et al. 2003) show that the colour segregation observed between local group and field galaxies is even seen at $z \sim 1$ (Cooper et al. 2006a,b; Gerke et al. 2006). DEEP2 is a spectroscopic survey of galaxies at redshifts around unity $(0.7 \lesssim z \lesssim 1.4)$, to a limiting magnitude of $R_{A B}=24.1$. The unprecedented combination of depth and redshift accuracy allows for an examination of the influence of the environment on the galaxies' properties at $z \sim 1$. Cooper et al. (2006b) use a sample of 19464 galaxies drawn from the DEEP2 survey to show that the colour-density relation evolves continuously, with red galaxies more strongly favouring overdense regions at lower redshift as compared to their highredshift counterparts, with the fraction of blue galaxies (which is lower in groups than in the field) staying roughly constant with redshift. However, at $z \simeq 1.3$, the red fraction starts to correlate only weakly with overdensity (Cooper et al. 2006a), and the group and field blue fractions become indistinguishable (Cooper et al. 2006b).

Cucciati et al. (2006) also carried out an investigation of the redshift and luminosity evolution of the colour-density relation using data from the VVDS (Le Fèvre et al. 2005), and also found that the trend for red(/blue) galaxies to be found mainly in dense(/underdense) regions seen at lower redshifts progressively disappears in the highest redshift bins investigated $(1 \lesssim z \leq 1.5)$.

Ilbert et al. (2006a) reconstructed the 3D density field using a Gaussian filter of smoothing length $5 \mathrm{~h}^{-1} \mathrm{Mpc}$, and estimated the luminosity function of 6582 galaxies of the VVDS in four redshift bins between $z=0.25$ and $z=1.5$, for galaxies in overdense and underdense environments, respectively. They find a strong dependence of the luminosity function on environment up to $z=1.2$, that is, a steeper slope in underdense regions, and a steepening with increasing redshift. In the redshift range $0.6 \leq z \leq 0.9$ they split the sample into red and blue galaxies, and again find the slope to be steeper in underdense regions, independent of spectral type. This is different from our results (where the slope of the luminosity function of blue galaxies remains unaffected by the local density, whereas for the red galaxies it changes), but their interpretation, albeit with a slightly different perception of the results, is compatible with ours: Together with the observations of
Cucciati et al. (2006), they interpret their result not as a lack of faint red galaxies in underdense regions, but as an increase of their number density in overdense regions with cosmic time.

This generally observed trend - the growing fraction of red galaxies in overdense regions, while the overall fraction of blue galaxies evolves slowly up to $z \simeq 1$ - suggests that the strong dependence of the galaxy properties on the environment found at lower redshifts is a result of environment-driven mechanisms. The build-up of the red sequence appears to have occurred preferentially in overdense regions. One further piece of evidence in this direction come from Gerke et al. (2006), who find that at $z \geq 0.7$ red galaxies already tend to be bright, and bright galaxies in general tend to live in dense environments, even at redshifts around unity.

Our results complement and reinforce this general picture, and fit into the standard model of hierarchical clustering growth and galaxy evolution: bright, massive galaxies formed early in the rare, highly clustered high-mass peaks of the dark matter distribution (Kaiser 1984) and are thus more strongly clustered than faint, less massive galaxies which have formed later in less clustered environments. But not only are bright galaxies more strongly clustered than faint ones, red galaxies are in addition much more strongly clustered than blue galaxies. It is commonly believed that the red, early-type galaxy population are remnants of merger processes, whereas the blue galaxies form stars at a rate only determined by their internal physical properties (Baldry et al. 2004; Bell et al. 2004), independent of their environment. In low-density regions, galaxies typically reside in the centres of low-mass dark matter haloes and are thus faint. Since there is still gas available for star formation, they are also blue. The merger rate is low, so they are mainly spirals. In higher density regions the typical galaxy is a central galaxy of a more massive dark matter halo, so it is tends to be bright. There is no gas left for star formation, and the merger rate was high, so such galaxies are rather red and early type. This interpretation is supported by the fact that the luminosity function of blue galaxies remains unchanged with the local density contrast, whereas the luminosity function of the red galaxies depends on the environment.

\section{Summary and outlook}

The dependence of galaxy properties on the environment in which they reside is a clue to the physical processes that led to their formation and present appearance: if the local density contrast changes the path the evolution of a galaxy takes (by merging, gas stripping, etc.), then this should be reflected in the properties of the galaxies that inhabit different environments. The means of investigating this correlation of galaxy properties and the local density contrast is the type- or colour-dependent luminosity function, calculated in different density regimes.

These measures, local overdensities and luminosity functions, make different demands on the data: For a precise determination of overdensities good redshift quality is needed, in the most optimal case spectroscopic. But current spectroscopic redshift surveys are either not deep enough or have too small statistics to allow for a precise measurement of the luminosity function, especially at intermediate to high redshifts.

In this paper, we have demonstrated how multicolour surveys can be used to overcome this problem. Multicolour surveys have larger redshift errors than spectroscopic ones, and redshift inaccuracies smooth out the structures. The extent to which the amplitudes are suppressed depends on the size of the redshift errors, but if the redshift error distribution is well determined, this can be taken into account. 
Red galaxies have - statistically - better redshifts than blue galaxies (because the redshift accuracy depends on magnitude, and red galaxies are statistically brighter than blue ones). So when we intend to compare their overdensities, we have to blur the good red galaxies' redshifts in order to make their redshift error distribution resemble that of the blue ones. The method, which makes use of the convolution theorem, was successfully tested with a mock COMBO-17 survey.

We have used the COMBO-17 survey to calculate overdensities for different samples of galaxies (a red, blue, bright and faint subsample, respectively), in three fields. In order to make the measurements comparable to each other, the redshifts of the subsample possessing the smaller redshift errors were blurred before calculating the overdensities. Instead of calculating the overdensity in small spheres, as is usually done, we do it in thin redshift slices. We find that one of the three COMBO-17 fields, the Chandra Deep Field South (CDFS), displays a relatively large underdense region, where the other two fields have overdensities fluctuating about mean density. We use this to compare the luminosity functions of red and blue galaxies in different density regimes (but at the same redshift, $0.25 \leq z \leq 0.4$ ).

The luminosity function of the blue cloud galaxies is unaffected by the environment: it has the same shape in all three fields. The luminosity function of the red sequence galaxies, on the other hand, is very different in the underdense region in the CDFS: its faint-end slope $\alpha$ is significantly more positive than in the other two fields at the same redshifts. This finding - that the underdensity is mainly due to a lack of faint red galaxies - is consistent with results at lower redshift (e.g. Croton et al. 2005 or Zandivarez et al. 2006), and fits into the common picture of hierarchical galaxy formation.

Our present analysis is only a preliminary study of how multicolour data can be used to investigate the dependence of galaxy properties on the local environment at redshifts $z \gtrsim 0.2$. A full quantification of the effect of the environment on galaxy properties will require much larger surveys. First of all the survey volumes have to be larger: not only will the statistics be better in a bigger survey, but also the dynamic range of observed overdensities. In COMBO-17, the range of overdensities that can be investigated is limited. In a large-area survey, the field can be split into many different smaller subfields (either randomly distributed or deliberately chosen by surface density) and a similar analysis to ours can be carried out, or a count-in-(large)-cells analysis similar to the one by Wild et al. (2005) and Conway et al. (2005), where they counted galaxies in approximately cubical boxes.

Second, a completeness to fainter magnitudes is desirable for a correct and precise determination of the slope $\alpha$ of the luminosity function also at higher redshifts. This is important for the investigation of the evolution of the dependence of galaxy properties on environment.

We can look forward to achieving many of these goals with new generations of deep multicolour or photometric redshift surveys, such as VST-16, KIDS (Kuijken 2006) or Pan-STARRS (Kaiser et al. 2005).

Acknowledgements. S. Phleps acknowledges financial support by the SISCO Network provided through the European Community's Human Potential Programme under contract HPRN-CT-2002-00316. J.A.P. was supported by a PPARC Senior Research Fellowship. CW was supported by a PPARC Advanced Fellowship. We would like to express our appreciation of the helpful discussions we had with Peter Schuecker, who sadly died in November 2006.

\section{References}

Adami, C., Mazure, A., Ilbert, O., et al. 2005, A\&A, 443, 805

Adelman-McCarthy, J. K., Agüeros, M. A., Allam, S. S., et al. (The SDSS Collaboration) 2006, ApJS, 162, 38

Baldry, I. K., Glazebrook, K., Brinkmann, J., et al. 2004, ApJ, 600, 681

Bell, E. F., Wolf, C., Meisenheimer, K., et al. 2004, ApJ, 608, 752

Bertin, E., \& Arnouts, S. 1996, A\&AS, 117, 393

Cole, S., \& Kaiser, N. 1989, MNRAS, 237, 1127

Bromley, B. C., Press, W. H., Lin, H., \& Kirshner, R. P. 1998, ApJ, 505, 25

Christlein, D., \& Zabludoff, A. 2003, ApJ, 591, 764

Colless, M. 1989, MNRAS, 237, 799

Colless, M., Dalton, G., Maddox, S., et al. (the 2dFGRS Team) 2001, MNRAS, 328, 1039

Colless, M., Bruce, A., Peterson, C. J., et al. (the 2dFGRS Team) 2003 [arXiv: astro-ph/0306581]

Conway, E., Maddox, S., Wild, V., et al. 2005, MNRAS, 356, 456

Cooper, M. C., Newman, J. A., Croton, D. J. et al. 2006a, MNRAS, 370, 198

Cooper, M. C., Newman, J. A., Coil, A. L., et al. 2006b

[arXiv: astro-ph/0607512]

Croton, D. J., Farrar, G. R., Norberg, P., et al. 2005, MNRAS, 356, 1155

Cucciati, Iovino, A., Marinoni, C., et al. 2006, A\&A, 458, 39

Davis, M., \& Geller, M. J. 1976, ApJ, 208, 13

Davis, M., \& Huchra, J. 1982, ApJ, 254, 437

Davis, M., Faber, S. M., Newman, J., et al. 2003, Proc. SPIE, 4834, 161

Dressler, A. 1980, ApJ, 236, 351

Dressler, A., Oemler, A., Couch, W. J., et al. 1997, ApJ, 490, 577

Faber, S. M., Willmer, C. N. A., Wolf, C., et al. 2006

[arXiv: astro-ph/0506044]

Fried, J. W., von Kuhlmann, B., Meisenheimer, K., et al. 2001,

Gerke, B. F., Newman, J. A., Faber, S. M., et al. 2006

[arXiv: astro-ph/0608569]

Gilli, R., Cimatti, A., Daddi, E., et al. 2003, ApJ, 592, 721

Gray, M. E., Wolf, C., Meisenheimer, K., et al. 2004, MNRAS, 347, 73

Ilbert, O., Cucciati, O., Marinoni, et al. 2006a

[arXiv: astro-ph/0602329]

Ilbert, O., Lauger, S., Buat, V., et al. 2006b, A\&A, 453, 809

Kaiser, N. 1984, ApJ, 284, L9

Kaiser, N., \& Pan-STARRS Team 2005, AAS, 37, 1409

Kuijken, K. 2006, online.kitp.ucsb.edu/online/lens06/kuijken/

Le Fèvre, O., Vettolani, G., Garilli, B., et al. 2005, A\&A, 439, 845

Lin, H, Kirshner, R. P., Shectman, S. A., et al. 1996, ApJ, 464, 60

Madgwick, D.S., Lahav, O., Baldry, I. K., et al. 2002, MNRAS, 333, 133

Marinoni, C., Giuricin, G., Costantini, B., Monaco, P., et al. 1999, in Observational Cosmology: The Development of Galaxy Systems, ed. G. Giuricin, M. Mezzetti, \& P. Salucci, ASP Conf. Ser., 176, 292

Martínez, H. J., Zandivarez, A., Merchán, M. E., et al. 2002, MNRAS, 337, 1441

Marzke, R. O., Geller, M. J., Huchra, J. P., et al. 1994, AJ, 108, 437

Meneux, B., Le Fèvre, O., Guzzo, L., et al. 2006, A\&A, 452, 387

Mo, H. J., \& White, S. D. M. 1996, MNRAS, 282, 347

Norberg, P., Baugh, C. M., Hawkins, E., et al. 2002, MNRAS, 332, 827

Phleps, S., \& Meisenheimer, K. 2003, A\&A, 407, 855

Phleps, S., Peacock, J. A., Meisenheimer, K., et al. 2006, A\&A, 457, 145

Ramella, M., Zamorani, G., Zucca, E., et al. 1999, A\&A, 342, 1

Ratcliffe, A., Shanks, T., Parker, Q. A., et al. 1998, MNRAS, 293, 197

Schmidt, M. 1968, ApJ, 151, 393

Sheth, R. K., \& Tormen, G. 1999, MNRAS, 308, 119

Trentham, N. 1998, MNRAS, 294, 193

Trentham, N., \& Tully, R. B. 2002, MNRAS, 335, 712

Valotto, C. A., Nicotra, M. A., Muriel, H., et al. 1997, ApJ, 479, 90

van Kampen, E., Jimenez, R., \& Peacock, J. A. 1999, MNRAS, 310, 43

van Kampen, E., Percival, W. J., Crawford, M., et al. 2005, MNRAS, 359, 469

Willmer, Faber, S. M., Koo, D. C., et al. 2006, ApJ, 647, 853

Wild, V., Peacock, J. A., Lahav, O., et al. 2005, MNRAS, 356, 247

Wolf, C., Meisenheimer, K., \& Röser, H. J. 2001a, A\&A, 365, 660

Wolf, C., Meisenheimer, K., Röser, H.-J., et al. 2001b, A\&A, 365, 681

Wolf, C., Meisenheimer, K., Rix, H.-W., et al. 2003, A\&A, 401, 73

Wolf, C., Meisenheimer, K., Kleinheinrich, M., et al. 2004, A\&A, 421, 913

Zandivarez, A., Martínez, H. J., Merchán, M. E., et al. 2006

[arXiv:astro-ph/0602405]

Zehavi, I., Zheng, Z. D. H., Weinberg, D., et al. 2005, ApJ, 630, 1

Zucca, Ilbert, O., Bardelli, S., et al. 2006, A\&A, 455, 879 